\title{
Nuevas combinaciones de aula inversa con just in time teaching y análisis de respuestas de alumnos
}

\section{New combinations of 'flipped classroom with just in time teaching' and learning analytics of student responses}

\author{
Alfredo Prieto Martin \\ David Díaz Martin \\ Isabel Lara Aguilera \\ Jorge Monserrat Sanz \\ Universidad de Alcalá (España) \\ Paquita Sanvicen Torner \\ Universitat de Lleida (España) \\ Raúl Santiago Campión \\ Universidad de la Rioja (España) \\ Alfredo Corell Almuzara \\ Universidad de Valladolid (España) \\ Melchor Álvarez-Mon Soto \\ Universidad de Alcalá (España)
}

\section{Resumen}

Este artículo revisa los procedimientos y resultados obtenidos con el método de aula inversa con 'just in time teaching' (JiTT) que sirve para conocer qué es lo que los alumnos no logran comprender en los materiales instructivos facilitados. Se han utilizado métodos de marketing y gamificación para lograr que los alumnos realicen el estudio previo y se han desarrollado métodos de análisis de las respuestas de los alumnos que permiten al profesor conocer los aspectos que les resultan más interesantes y difíciles de comprender, así como sus dudas más urgentes. Se han desarrollado métodos para usar las dudas de los alumnos para generar retroinformación formativa y actividades para realizar en clase. En el método denominado "Aprendizaje inverso fuerte" el profesor contesta proporcionando respuestas a las dudas urgentes de todos sus alumnos por correo electrónico. En el método denominado "Da la vuelta en colores" se clasifican las dudas de los alumnos en función de su posible utilidad en clase utilizando un código de colores. La aplicación combinada de estos métodos 
ha logrado reducir la tasa de fracaso de los estudiantes, aumentar su calificación media en las pruebas de evaluación del aprendizaje en más de una desviación estándar y la tasa de alumnos que superan el nivel de maestría ha aumentado. También han mejorado las valoraciones de los alumnos sobre la docencia de estas asignaturas. Finalmente, se discuten las razones por las que esta combinación de métodos es tan eficaz.

Palabras clave: aprendizaje semipresencial; aula inversa; enseñanza justo a tiempo; aprendizaje inverso fuerte; da la vuelta en colores; gamificación.

\begin{abstract}
The results obtained thanks to the flipped classroom with 'just Iiin time' teaching method are reviewed. This method allowed to know what the students did not understand after trying to study the instructive materials assigned to them. To achieve that the students work before class methods of marketing and gamification were developed. Methods for the analysis of student responses were also developed. The JiTT method allowes the teacher know in advance those aspects that are most interesting or most dificult to understand for the students as well as their most urgent doubts. Finally, we have developed methods to use the urgent doubts of the students to generate formative feedback and activities to do in the classroom. In the method named "flipped learning forte" the teacher answer the urgent doubts of his students. In the method named 'flip in colours' the teacher classify the doubts by their posible utility in the classroom using a color code. With the combined application of these methods in university courses the failure rate of the students has decreased and the mean grade in the exams for the assessment of learning has increased in more than one standard deviation. The rate of students that attain the level of mastery has increased, as well as the student evaluations about the teachers of these courses. Finally, the reasons underlying the efficacy of the proposed flipped method are discussed.
\end{abstract}

Keywords: blended learning; flipped classroom; just in time teaching; flipped learning forte; flip in colours; gamification.

El flipped classroom o aula inversa implica transmitir a los alumnos la información a aprender por medios electrónicos para evitar tener que consumir en su explicación la mayor parte del tiempo de clase (Sams, 2012). El tiempo así ahorrado es empleado en actividades de aula en las que el alumno es el protagonista activo. El flipped classroom reduce el tiempo de instrucción directa y prepara a los alumnos para discutir ejemplos o supuestos prácticos y realizar, bajo la atenta supervisión del profesor, actividades de mayor nivel cognitivo como analizar, evaluar y crear conocimiento (Bloom, 1956; Churches, 2007) a partir de la información previamente aportada y asimilada por el discente (Touron, 2014). El flipped classroom es un primer paso en una senda de cambio educativo que permite a continuación progresar hacia un aprendizaje más centrado en el protagonismo del alumno, su autonomía y su autorregulación (Sams, 2014). 
La analítica de resultados de aprendizaje es el proceso de recopilación, estructuración y análisis de datos en los procesos de enseñanza y aprendizaje utilizando tecnologías de la información y la comunicación (Amo, 2016). En este trabajo mostramos maneras de obtener información sobre el aprendizaje de los alumnos tras las actividades de preparación previas a la clase. También se discuten procedimientos para el análisis de sus respuestas que permitirá a los profesores decidir qué actividades será prioritario realizar en el aula para lograr que los alumnos comprendan los conceptos esenciales.

Los autores de este trabajo se basaron en un modelo de aprendizaje centrado en el alumno (Weimer, 2013) que se fundamenta en que los alumnos aprenden más y mejor cuando sus profesores tienen un conocimiento real sobre su nivel de comprensión inicial, y pueden por tanto desafiar con ejemplos concretos sus conceptos erróneos de partida y construir su comprensión a partir de aquello que ya comprenden bien (Huston, 2012). Para crear este modelo, optamos por usar una metodología de aprendizaje semipresencial (blended learning) la enseñanza justo a tiempo (just in time teaching, JiTT) en la que la comunicación bidireccional entre profesores y alumnos permite crear este ambiente de aprendizaje ideal en el que el docente tiene conocimiento de las dificultades e intereses de sus discentes (Novak, 1999).

Se ha desarrollado un método que combina el JiTT con flipped classroom y elementos de gamificación que se ha denominado "aula inversa con enseñanza justo a tiempo" (flipped classroom with just in time teaching, FC/JiTT) (Prieto, 2014). En este método, se utilizan cuestionarios de comprobación del estudio previo con preguntas de respuesta libre que hacen reflexionar a los alumnos e informan al profesor de sus reacciones tras la interacción con los materiales didácticos. Las respuestas de los alumnos se analizan y se obtienen conclusiones que permiten a los profesores conocer antes de empezar las clases qué es lo que a sus alumnos les cuesta más comprender en los materiales instructivos facilitados (Prieto, 2014). Por tanto, el FC/JiTT primero permite detectar los problemas de comprensión más prevalentes entre los alumnos en los temas tratados en las asignaturas. Esta información aporta indicaciones sobre cómo preparar nuevos materiales instructivos mejorados, actividades y ejemplos que ayuden a que se comprendan mejor esos conceptos.

El FC/JiTT fomenta que los alumnos sean responsables de su aprendizaje inicial y adopten un abordaje profundo orientado a la comprensión en profundidad de la materia a tratar (Biggs, 2006; McTighe y Wiggins, 2013). El análisis de las respuestas de los alumnos ofrece respuestas a preguntas muy relevantes sobre lo que les interesa más y lo que no logran comprender tras interaccionar con los materiales instructivos proporcionados.

Los autores de este artículo, miembros activos del registro de profesores que usan el modelo de aprendizaje inverso en países de habla hispana (Prieto y Vélez, 2016) y del grupo de innovación docente universitaria magistrales anónimos, proponen en este trabajo combinar el modelo de aprendizaje inverso con el análisis de respuestas 
de los alumnos a cuestionarios para así adaptar las actividades de clase y optimizar el aprendizaje de los alumnos. Con el método propuesto el profesor recoge en respuestas a cuestionarios on line las reacciones de sus alumnos tras su interacción con los materiales (informe del alumno), las analiza rápidamente para obtener información que será útil para replantear sus actividades de enseñanza, aprendizaje y evaluación formativa en el aula. Este análisis justo a tiempo del punto de vista de los alumnos tras interaccionar con los materiales instructivos genera conclusiones sobre lo que más les interesa y les parece más novedoso y también sobre lo que más les cuesta comprender. Esta información puede ser utilizada para decidir nuevas actividades que pueden ayudar a nuestros alumnos a superar sus dificultades de aprendizaje.

En el primer año de implementación de la metodología FC/JITT, se crearon materiales instructivos para transmitir la información a los alumnos y se intentó detectar e interpretar sus dificultades y reaccionar a sus respuestas modificando al modo just in time los planes de clase introduciendo nuevos materiales y explicaciones en clase. En el segundo año de implementación, se fueron añadiendo más materiales instructivos en vídeo para aclarar aquellos conceptos que resultaban ser más difíciles de comprender para los alumnos y se desarrollaron herramientas de evaluación formativa destinadas a hacer aflorar esos conceptos con los que se producen los mayores problemas de comprensión. También se incorporaron las metodologías peer instruction (Mazur, 1997) y team based learning (Michaelsen, 2002) y se gamificaron las asignaturas incorporando un sistema de pequeñas recompensas para incentivar el esfuerzo y el estudio previo de los alumnos a cambio de pequeñas bonificaciones en la calificación (Chou, 2015; Markzewski, 2013a).

En años posteriores la forma de utilizar las respuestas de los alumnos para proporcionarles feedback y crear actividades para clase ha ido evolucionando. Primero se ideo y desarrolló el método aprendizaje inverso fuerte (flipped learning forte) que consiste en responder a la duda urgente que cada alumno ha planteado. En los dos últimos años se ha desarrollado un nuevo método denominado "Da la vuelta en colores" (flip in colours) en la que el profesor clasifica y colorea en distintos colores las dudas urgentes de los alumnos en función de su tipología y posibles usos didácticos. Unas dudas se usan para generar debates o pedir a algunos alumnos que las expliquen a sus compañeros, otras las aclara el mismo profesor. Otras se usan como punto de partida de proyectos de indagación. Adicionalmente, cada duda se etiqueta por su temática y se ordenan usando estas etiquetas para así agruparlas temáticamente y saber qué momento de la clase es el más apropiado para tratarlas.

\section{FASES EN LA IMPLEMENTACIÓN DEL FC/JITT}

A continuación, se explica cómo implementar una serie de elementos que a juicio de los autores son críticos para la implementación exitosa del FC/JiTT. 


\section{Motivación del compromiso de los alumnos con el estudio previo}

El éxito de los métodos de aula inversa depende de que el docente logre que los alumnos realicen estudios y actividades de preparación para las clases presenciales. Para conseguir este fin es necesario concienciar en la presentación de la asignatura a los alumnos para que estudien antes de las clases. Esta concienciación deberá reforzarse con sistemas de recompensa a cambio de acciones (gamificación) y con acciones del profesor que demuestren a los alumnos que los profesores estudian meticulosamente sus respuestas y generan feedback a partir de ellas.

\section{Presentación del método de aula inversa a los alumnos al inicio de la asignatura}

En la clase de presentación de la asignatura se pretende que los alumnos encuentren motivos para que se comprometan con la realización del estudio previo antes de cada tema. Se debe lograr que los alumnos decidan empezar a hacer el estudio previo del primer tema y después que sigan haciéndolo en los siguientes. Para ello, será necesario realizar en la presentación inicial de la asignatura una demostración de las ventajas del método para los alumnos que realizan el estudio preparatorio. También es conveniente proporcionar refuerzo positivo de su trabajo día a día reconociendo públicamente y con bonificaciones en calificación el esfuerzo de preparación y proporcionando a los alumnos retroinformación a sus expectativas, intereses y necesidades mostradas en sus respuestas a los cuestionarios.

Para ello, les planteamos a nuestros alumnos en el primer día de clase un ejercicio en el que deben identificar que distribuciones de notas corresponden a distintos tipos de asignatura: una asignatura "María" (en la que es fácil sacar buena nota), una "ruleta rusa" (en la que te puede ir bien o te puede ir muy mal) y una "hueso" (en la que es muy difícil aprobar) (figura 1). Les pedimos supongan que son tres asignaturas optativas y que decidan en cuál de las tres desearían matricularse. Suelen responder de manera unánime que preferirían matricularse en la asignatura "María" (columna de la derecha en la imagen) para así sacar mejores calificaciones. Seguidamente, se les desvela que las tres distribuciones de notas mostradas en la figura 1 son de la misma asignatura, pero que corresponden a tres subgrupos de alumnos que respondieron de distinta manera al modelo de aprendizaje inverso. El subgrupo que realizó el estudio previo en la mayoría de los temas obtuvo excelentes resultados (columna derecha asignatura supuestamente más fácil), el de los alumnos que efectuaron el estudio previo de manera ocasional y esporádica obtuvo peor nivel (columna central asignatura de dificultad intermedia) y el grupo de los que nunca realizaron el estudio previo consiguió unos resultados lamentables (columna izquierda asignatura difícil de aprobar). 
Figura 1. Ejemplo de ejercicio a realizar en la presentación de una asignatura para motivar a los alumnos a comprometerse con el estudio de los temas antes de que sean tratados en clase. Se pide a los alumnos que determinen cuál de las asignaturas es una María, cual un hueso y cual una ruleta rusa. Después de que lo hagan se desvela el misterio. Las tres columnas A, B y C corresponden a subgrupos de alumnos de la misma asignatura que usa el método FC/JiTT. La de la izquierda (A) son las calificaciones de los alumnos que nunca hicieron el estudio previo, la central (B) las calificaciones de los que solo estudiaron en una minoría de ocasiones y la de la derecha (C) las calificaciones del subgrupo de alumnos que realizo el estudio previo en la mayoría de los temas.

\section{Eercicio de motivación en la presentación de la asignatura}

En función de la distribución de notas de cada asignatura decide cual es

- Una María

- Una ruleta rusa

- Un Hueso

Si fuesen tres asignaturas optativas ... ¿Cuál escogerías?

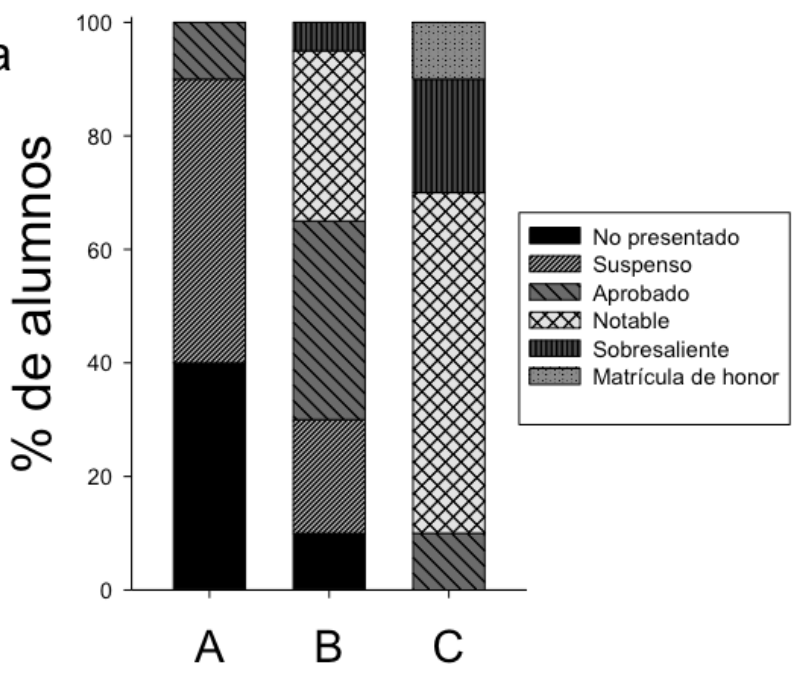

Manteniendo la motivación de los alumnos a lo largo de todo el periodo de impartición de la asignatura

Además del marketing en el primer día de clase, otras medidas fomentan que los alumnos se comprometan con el estudio previo (figura 2). Estas incluyen anunciar que el estudio previo y la participación en clase serán bonificadas en la calificación de la asignatura (gamificación con pequeñas recompensas a cambio de pequeñas acciones) (Chou, 2015). También se aplica la gamificación mediante la apelación al sentido de misión y se les demuestra como aceptar un mayor grado de responsabilidad sobre su propio aprendizaje les ayudará a desarrollar competencias para el aprendizaje autónomo y autorregulado (Chou, 2015; Markzewski, 2013b). También se les entregan en la primera clase cartas de compañeros de promociones anteriores que sacaron sobresaliente o matrícula de honor, en las que les cuentan cuál fue la estrategia de estudio y trabajo que les llevó al éxito en la asignatura. 
Además de estas acciones de marketing inicial es importante proporcionar feedback día a día a nuestros alumnos sobre su trabajo de estudio previo.

Figura 2. Cómo podemos motivar a nuestros alumnos para que estudien los temas antes de que sean tratados en clase

\section{En el diseño y presentación de la asignatura (día D)}

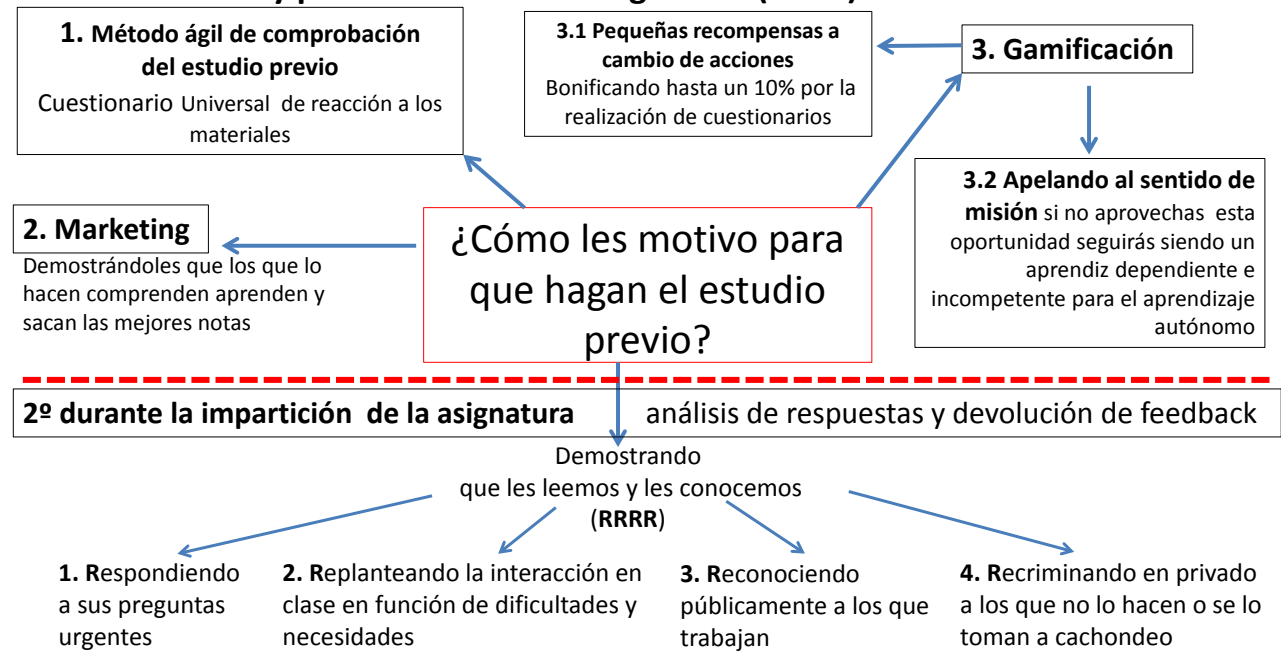

\section{Elaboración de cuestionarios de comprobación del estudio previo}

Para ello utilizamos la aplicación gratuita Google Forms. El cuestionario debe incluir preguntas que fuercen a reflexionar sobre lo que ha comprendido y lo que no. Google forms recopila las respuestas en una hoja de datos (Google Spreadsheet).

Ejemplo de un cuestionario muy completo, aunque a los principiantes en el uso de esta metodología les recomendamos empezar por cuestionarios más sencillos de cinco a diez preguntas:

1. Apellidos y nombre.

2. ¿Cuál te parece la idea más importante o interesante que has aprendido en este tema y justifica por qué?

3. ¿Qué parte del tema te parece más importante profundizar en clase y justifica por qué?

4. Resume lo más importante del tema en cien palabras.

5. ¿Qué es lo que no consigues comprender o te queda menos claro, explica cuál es tu dificultad? 
6. ¿Qué pregunta o duda urgente te gustaría que respondiéramos el primer día?

7. ¿Qué parte del tema no necesitas que te expliquen? ¿Por qué?

8. ¿Qué has conseguido aprender o comprender al ver los vídeos?

9. Pon aquí tu dirección de email por si tenemos que pedirte que estudies más.

10. ¿Sobre qué cuestión relativa a este tema te gustaría profundizar e indagar por tu cuenta?

11. Si al estudiar crees que has comprendido algo importante y que al principio no comprendías. Explica que es lo que has comprendido y propón una pregunta que otros compañeros que también lo han comprendido puedan responder.

12. Haz una crítica constructiva de los materiales instructivos facilitados e indica qué crees que se podría mejorar en los materiales para que los entendieses mejor y con más claridad.

13. ¿Cuánto tiempo has tardado en leerte los materiales de los pdfs de las diapositivas del tema?

14. ¿Cuánto tiempo has tardado en ver los vídeos y leerte las transcripciones del audio en inglés?

15. ¿Cuánto tiempo has tardado en contestar a este cuestionario?

A este cuestionario universal, le podemos añadir preguntas específicas sobre la información contenida en el tema. Esto posibilitará la evaluación automática de estas preguntas y nos permitirá detectar qué preguntas son las que más fallan tras el estudio de los materiales. Esta metodología de evaluación online tras el estudio previo, pero antes de la clase se denomina PEPEOLA (preparación y estudio previo por evaluación online automática (Robles, 2010).

El cuestionario más minimalista en educación universitaria debería contener al menos las preguntas para identificar al alumno, saber lo que ha comprendido, lo que no ha conseguido comprender y el tiempo de trabajo que ha invertido en leer materiales, ver vídeos y responder al cuestionario.

\section{Análisis de respuestas de los alumnos a los cuestionarios}

Desde Google forms exportas o copias y pegas a Excel la matriz de respuestas a las preguntas del cuestionario de comprobación del estudio previo. En un libro de Excel las respuestas al cuestionario se descomponen o "deconstruyen" en varias hojas:

1. Aspectos más importantes y novedosos.

2. Aspectos que no se entienden y dudas urgentes: analíticas de dudas.

3. Qué les han ayudado a comprender los vídeos y las transcripciones de audio.

4. Contabilidad de carga de trabajo temporal para los alumnos.

5. Apellidos y nombres para la contabilidad de bonificaciones. 
¿Cómo responder al conocimiento sobre los conceptos que resultan oscuros ('muddiest points')?

Con las respuestas a la pregunta: ¿Qué es lo que no está claro tras el estudio y permanece difícil de comprender? Creamos el ranking o clasificación ordenada de los tópicos o conceptos que resultan más oscuros o problemáticos para nuestros alumnos (Angelo y Cross, 1993). Para realizar este tipo de análisis (figura 2) primero se examinan las respuestas y se eligen etiquetas para categorizar las respuestas e identificar, por ejemplo, los tópicos que los alumnos consideran más difíciles de comprender, interesantes, novedosos o valiosos. Al lado de la columna de las respuestas se crea una nueva columna para colocar la etiqueta de cada respuesta a su lado, de esta manera se puede categorizar la diversidad de las respuestas de los alumnos. Una vez que se etiquetan todas las respuestas se pueden ordenar usando como criterio la etiqueta asociada a cada respuesta. Se ordenan alfabéticamente el bloque de celdas usando como criterio la columna de las etiquetas y ya tenemos ordenadas las preguntas de nuestros alumnos. Esto nos permite juntar todas las respuestas relacionadas con un concepto o tema y así podemos tratarlas cuando lleguemos a esa parte del tema, ponemos más ejemplos y ejercicios sobre los temas que más interesan o explicamos más a fondo aquello que les cuesta más comprender. También podemos cuantificar cuantos alumnos señalan cada tópico e incluso representar en forma de gráficos el ranking de conceptos más difíciles en clase.

Figura 3. Se ilustran los pasos a dar para la codificación y categorización de respuestas de los alumnos en Microsoft Excel

\section{Proceso de análisis de respuestas: 1. ensanchado, 2. etiquetado,} 3. ordenación, 4. cuantificación de lo que no se entiende

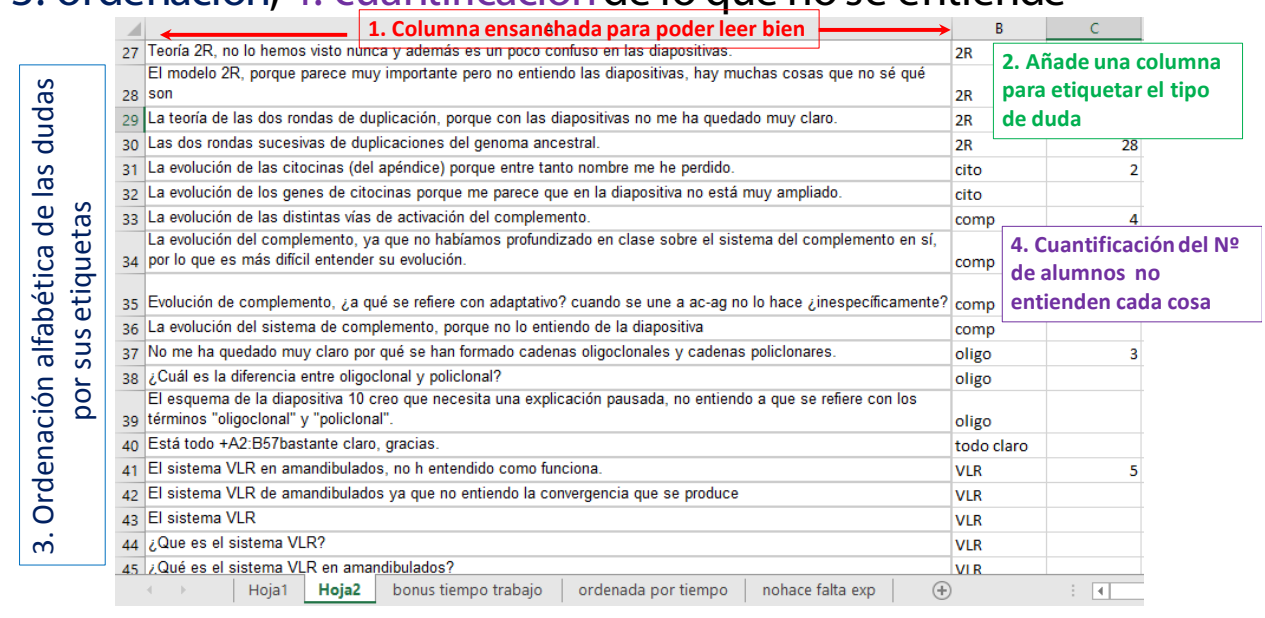


Esta retroinformación ayudará a que los alumnos tomen conciencia de cuáles son los conceptos más difíciles de comprender para sus compañeros. Al trabajar de esta manera en clase, además enviamos el meta-mensaje de que nos interesa conocer sus intereses y que respondemos a ellos alterando nuestra docencia y que a cambio de su trabajo de estudio previo les damos poder para influir en lo que se tratará en clase.

Identificación de los alumnos que estudian y contabilización de las bonificaciones que merecen por su trabajo de estudio preparatorio

La elaboración de listas de los alumnos que hicieron el estudio previo es muy sencilla. Se copia y pega la columna de nombres en una nueva hoja, se ordena alfabéticamente e insertan celdas vacías en los lugares ocupados por los alumnos que no hicieron la tarea. Solo restará copiar y pegar esta columna en la lista de calificaciones para así asignar bonificaciones a aquellos alumnos que hicieron el trabajo de preparación.

Existen distintos procedimientos para la evaluación formativa en los métodos de fomento del estudio previo. En el FC/JiTT el procedimiento es muy sencillo solo se requiere revisar las respuestas y elaborar la lista de alumnos que respondieron cada cuestionario y superaron un control de calidad mínima en sus respuestas. A los que no superan este control, pues sus respuestas demuestran que no han abordado el estudio con un nivel mínimo de profundidad, se les puede enviar un e-mail urgente dándoles una segunda oportunidad.

En las metodologías que comprueban el estudio previo mediante cuestionarios de preguntas de elección entre múltiples opciones (team based learning, peer instruction, PEPEOLA) es conveniente registrar las respuestas de los alumnos a nuestras preguntas de evaluación formativa. Esto puede hacerse con Apps gratuitas como Socrative o Google forms y también con las herramientas a tal efecto de la plataforma de e-learning de la institución (Moodle o Blackboard). Para aumentar la participación en clase es conveniente registrar en una lista a los alumnos que participen en clase.

\section{Cálculo de la carga de trabajo correspondiente al estudio previo}

Los profesores tendemos a subestimar las demandas de tiempo de las tareas que mandamos para casa y no somos capaces de anticipar las dificultades que nuestros alumnos tendrán con las tareas (Bain, 2004; Prieto, 2006; Svinicki, 2004). Si no obtenemos una medida realista del tiempo que exige la realización de las tareas a nuestros alumnos es posible que les sobrecarguemos de trabajo y esto tenga un efecto negativo sobre su motivación. Si nuestra evaluación es demasiado exigente y los que trabajan no se ven recompensados eso afectará negativamente a su 
motivación por seguir trabajando en nuestra asignatura. Por todo ello, es importante calcular el tiempo de trabajo de los alumnos en el estudio previo. Para ello añadimos una columna de etiquetas en la que vamos apuntando los tiempos de trabajo de cada alumno en forma de números en minutos (para que la hoja de cálculo pueda procesarlos matemáticamente).

Esto nos permitirá calcular fácilmente la carga de trabajo que ocasionan las tareas de preparación para el aprendizaje inverso. Nuestros estudios de carga de trabajo para el alumno demuestran que el tiempo medio semanal dedicado al estudio previo de una asignatura no supera las dos horas de trabajo y por tanto entra dentro del volumen de carga de trabajo exigible a un alumno universitario. Dado que los estudios de carga de trabajo real en universidades españolas indican que esta es inferior a la nominal de los créditos ECTS (Barjola, 2011; Gómez-Esquer, 2011) la adición del modelo inverso con su requerimiento de estudio previo puede ayudar a que la carga de trabajo de nuestros alumnos se acerque algo más a la carga de trabajo nominal ideal de sus asignaturas establecida por los créditos ECTS (Prieto, 2014b).

Diversos modos para usar las respuestas de los alumnos para generar nuevos materiales ('just in time teaching'), feedback ('flipped learning forte') y actividades ('flip in colours')

Lo más sencillo es tomar nota de algunas de las preguntas urgentes para responderlas en clase, pero disponemos de otras opciones metodológicas más sofisticadas para el tratamiento de las dudas urgentes de los alumnos. En el just in time teaching a partir de las respuestas de nuestros alumnos decidimos qué nuevos materiales instructivos es necesario crear para solventar y aclarar las dudas más frecuentes entre los alumnos. También se pueden crear viñetas experimentales o estudios de casos que ilustren y trabajen los conceptos que están siendo más difíciles de comprender para los alumnos. En el flipped learning forte, el profesor contesta a las dudas urgentes de los alumnos y les hace llegar sus respuestas a una lista de e-mail que incluye a todos los alumnos (incluso a los que no se molestaron en realizar el estudio preparatorio). Si la clase está formada por muchos alumnos estas respuestas deberán ser muy concisas, casi telegráficas.

Alternativamente, podemos clasificar las dudas urgentes en varios tipos: las que requieren una explicación del profesor, las que requieren una investigación o indagación por parte del profesor, las que pueden ser respondidas por compañeros con un mejor nivel de comprensión, las que ponen en evidencia alguna concepción errónea de partida. También se puede seleccionar algunas para discutir en clase o para que las indaguen los alumnos por su cuenta. Finalmente, podemos señalar a nuestros alumnos aquellas de sus preguntas que, algunas de las cuales pueden inspirar probables preguntas de examen. Es seguro que eso atraerá su interés y les hará estudiarlas. Estos tres modos de usar las respuestas de los alumnos dan 
lugar a distintos métodos de implementación del modelo inverso que se tratarán a continuación.

\section{VARIANTES METODOLÓGICAS PARA IMPLEMENTAR EL MODELO INVERSO EN ASIGNATURAS UNIVERSITARIAS}

A continuación, vamos a mostrar en más detalle tres métodos que hemos utilizado para implementar el modelo inverso con nuestros alumnos de grado: el flipped classroom with just in time teaching, el flipped learning forte y el flip in colours.

\section{Flipped classroom with just in time teaching (aula inversa con enseñanza justo a tiempo)}

El método más sencillo para el trabajo con dudas urgentes es que el profesor se las imprima, señale aquellas que podrían comentarse y las lleve a clase donde las comentará y responderá. El profesor puede enfocar la clase en la resolución de esas dudas: plantea ejercicios para trabajarlas, crea nuevos materiales, ejemplos y ejercicios destinados a esclarecerlas. Al emplear este método los alumnos perciben que su profesor se preocupa y se esfuerza en ayudarles a comprender precisamente aquello que más les cuesta aprender.

\section{Flipped learning forte}

En este método se envía a los alumnos las respuestas concisas a sus preguntas urgentes. Esto lo hacemos respondiendo en una lista de las dudas urgentes a todos los alumnos. A continuación, enviamos esta lista de las respuestas a las dudas a todos nuestros alumnos antes de que el tema sea tratado en clase y se les recomienda que se las miren y estudien (y que posteriormente las repasen antes de las actividades de evaluación formativa y acreditativa). Para implementar de manera segura este procedimiento es recomendable cortar y pegar la columna desde el spreadsheet de Google forms a Microsoft Excel donde se responde a las preguntas y así se evita dañar sin querer el spreadsheet original. Se añade en Excel al lado derecho de la columna de las dudas urgentes, una nueva columna de respuesta a las mismas donde las contestamos con respuestas muy concisas. Para mejorar el formato de nuestras respuestas es recomendable copiar nuestra tabla de dudas y respuestas desde Excel a Word, pues en este programa podemos controlar mejor el formato de impresión de la tabla y además tiene mejor corrector ortográfico y se pueden insertar links a recursos suplementarios automáticamente. Cuando se emplea esta metodología para convertir las dudas de los alumnos en feedback formativo es conveniente responder 
en rojo a la duda de cada uno y marcar los links a recursos suplementarios en otro color distinto.

Finalmente, se envían a todos los alumnos las respuestas de su propia duda y las de todos sus compañeros. A esta lista de dudas urgentes respondidas por el profesor se debe añadir la indicación de que algunas de estas preguntas pueden ser útiles para las pruebas de evaluación, razón por la que se recomienda a los alumnos el revisarlas y estudiarlas cuidadosamente.

\section{Flip in colours}

En esta metodología las dudas de los alumnos se utilizan como inspiración para generar actividades en clase. Esto se logra mediante la clasificación de las dudas urgentes de los alumnos en varias categorías. Para aplicar este método de análisis en la hoja de respuestas de los alumnos a los cuestionarios se selecciona la columna de las dudas urgentes y se clasifican en varias categorías que se identifican coloreando su texto con distintos colores. Aquellas dudas que deben ser respondidas por el profesor se colorean en negro. Aquellas que deben ser indagadas por el profesor se señalan con un fondo amarillo. Aquellas que pueden ser respondidas por los alumnos se colorean en verde. En rojo se colorean aquellas que ponen en evidencia errores de conceptos básicos que deben ser señalados y esclarecidos por el profesor o los compañeros. En azul se colorean aquellas que pueden ser buenas preguntas de examen. En rosa aquellas que pueden servir para originar debates en clase. En naranja, aquellas que pueden originar indagación por los alumnos y en morado aquellas que nos dan pie a proponer nuevos recursos, ejemplos y ejercicios. 
Tabla 1. Clasificación de las dudas en el flip in colours en función de su posible uso en clase

\begin{tabular}{|l|l|l|}
\hline \multicolumn{1}{|c|}{ Clasificación de dudas } & \multicolumn{1}{|c|}{$\begin{array}{c}\text { Color del } \\
\text { texto }\end{array}$} & \multicolumn{1}{c|}{ Uso didáctico } \\
\hline $\begin{array}{l}\text { Las que requieren una } \\
\text { explicación por el profesor. }\end{array}$ & Negro & $\begin{array}{l}\text { Se ordenan temáticamente y las explica el } \\
\text { profesor cuando toque esa parte del tema. }\end{array}$ \\
\hline $\begin{array}{l}\text { Las que requieren una } \\
\text { indagación por el profesor. }\end{array}$ & $\begin{array}{l}\text { Negro con } \\
\text { fondo amarillo }\end{array}$ & $\begin{array}{l}\text { Las indaga el profesor y luego las explica o } \\
\text { usa de otra manera en clase. }\end{array}$ \\
\hline $\begin{array}{l}\text { Las que pueden ser explicadas } \\
\text { por un compañero con } \\
\text { comprensión más avanzada. }\end{array}$ & verde & $\begin{array}{l}\text { El profesor reenvía una lista de estas a } \\
\text { los alumnos a fin de que se preparen para } \\
\text { explicarlas en clase. Los voluntarios para } \\
\text { explicar serán bonificados. }\end{array}$ \\
\hline $\begin{array}{l}\text { Las que implican una } \\
\text { confusión o concepto erróneo } \\
\text { del alumno que las plantea. }\end{array}$ & Rojo & $\begin{array}{l}\text { El profesor señala la confusión y la } \\
\text { aclara o pide a los alumnos que intenten } \\
\text { descubrirla por sí mismos. }\end{array}$ \\
\hline $\begin{array}{l}\text { Las que pueden dar pie a } \\
\text { buenas preguntas de examen. }\end{array}$ & azul & $\begin{array}{l}\text { Se entrena en clase como responder a } \\
\text { preguntas de este tipo pues podrán caer } \\
\text { otras similares en el examen. }\end{array}$ \\
\hline $\begin{array}{l}\text { Las que pueden dar pie a } \\
\text { buenas discusiones en clase o } \\
\text { en el foro. }\end{array}$ & rosa & $\begin{array}{l}\text { Se proponen para discusión, las } \\
\text { aportaciones se valorarán. }\end{array}$ \\
\hline $\begin{array}{l}\text { Las que pueden originar } \\
\text { actividades o proyectos de } \\
\text { indagación. }\end{array}$ & Naranja & $\begin{array}{l}\text { Se proponen actividades de indagación o } \\
\text { investigación a partir de ellas. }\end{array}$ \\
\hline $\begin{array}{l}\text { Las que indican la necesidad } \\
\text { de añadir nuevos recursos } \\
\text { ejemplos o ejercicios. }\end{array}$ & Morado & $\begin{array}{l}\text { Se crean nuevos recursos ejemplos y } \\
\text { ejercicios. }\end{array}$ \\
\hline
\end{tabular}

\section{IMPACTO DE LOS MÉTODOS DE FOMENTO DEL ESTUDIO PREVIO SOBRE EL APRENDIZAJE DE LOS ALUMNOS}

En cursos posteriores este sistema de fomento del estudio previo se ha continuado optimizando y ajustando por medio de la incorporación de recursos instructivos y respondiendo a las dudas urgentes de los alumnos. Esto ha resultado en que los resultados de los alumnos han seguido mejorando progresivamente al ir aumentando los porcentajes de alumnos que realizan el estudio previo de los temas (figura 4). Este aumento progresivo y correlativo en las sucesivas promociones de los porcentajes de alumnos que realizan el estudio previo de más de la mitad de los temas (fila superior de gráficos) y las calificaciones medias obtenidas en los exámenes de evaluación del aprendizaje (fila inferior) muestra la estrecha relación la frecuencia con la que los alumnos de una clase realizan el estudio de preparación y la mejora en las calificaciones en las pruebas de evaluación del aprendizaje. 
Figura 4. Impacto del fomento del estudio previo sobre el aprendizaje. Asociación entre los porcentajes de alumnos que realizan el estudio previo en más de la mitad de los temas (fila superior) y la calificación media en las pruebas de evaluación del aprendizaje obtenida en tres asignaturas del grado en biología sanitaria en las que hemos implementado el modelo de flipped learning (fila inferior)
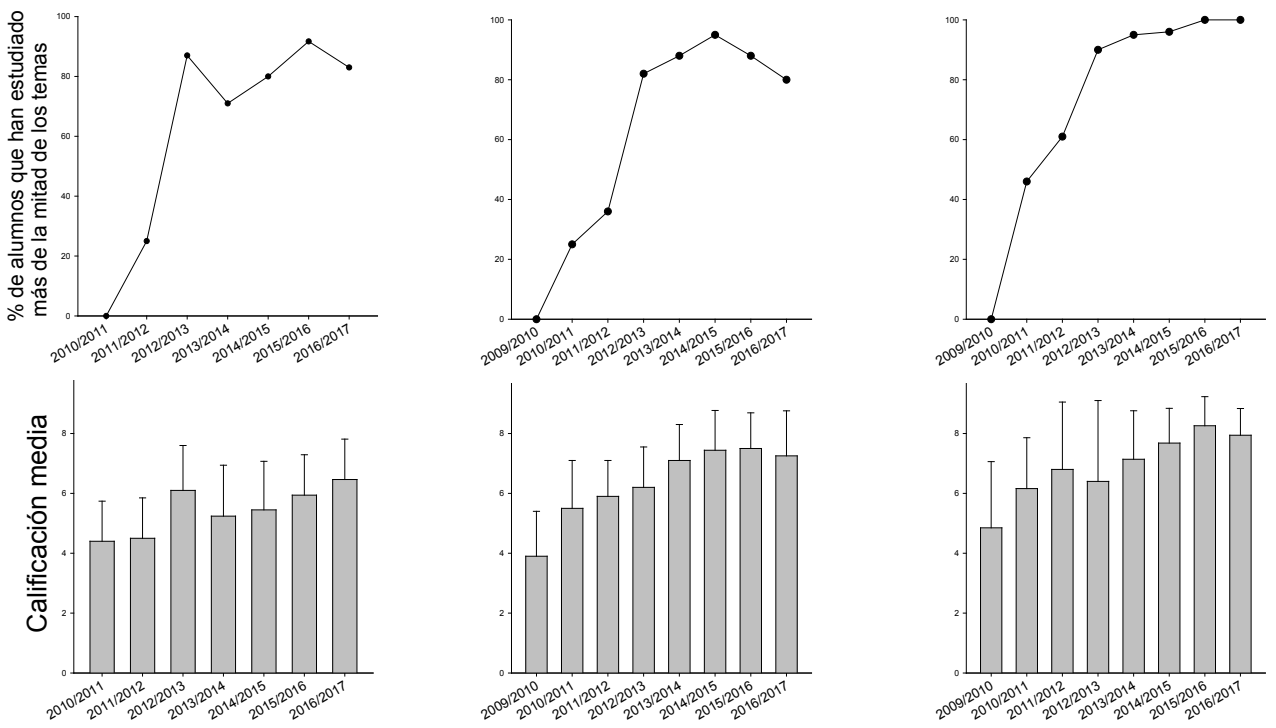

En los tres últimos cursos mostrados en las gráficas hemos utilizado nuevos métodos para proporcionar más y mejor feedback a nuestros alumnos sobre sus dudas urgentes (flipped learning forte y flip in colours). El uso combinado de estas dos metodologías de reacción del docente a las dudas de los alumnos ha permitido mejorar todavía más las calificaciones obtenidas por los alumnos en las pruebas de evaluación del aprendizaje (figura 4).

Las diferencias entre las últimas promociones con metodología expositiva tradicional y una promoción en la que combinamos las metodologías flipped learning forte y flip in colours se muestran en la figura 5. Como puede observarse la nota media aumenta, se reduce el porcentaje de alumnos que obtienen nota inferior a 5 y aumenta espectacularmente el porcentaje de alumnos que alcanzan el nivel de maestría, establecido en una puntuación igual o superior a 8 en las pruebas de evaluación del aprendizaje. Mejoras en calificaciones y reducción de tasas de fracaso han sido reportadas por otros autores al incorporar aprendizaje activo a sus clases (Freeman, 2014; Knight y Woods, 2005; Mazur, 1997; Michael, 2006; Prince, 2004). 
Figura 5. Curvas de frecuencia de alumnos que obtienen cada calificación en promociones de alumnos que han aprendido la asignatura mediante métodos expositivo tradicional y la combinación de los métodos de flipped learning de segunda generación flipped learning forte y flip in colours.

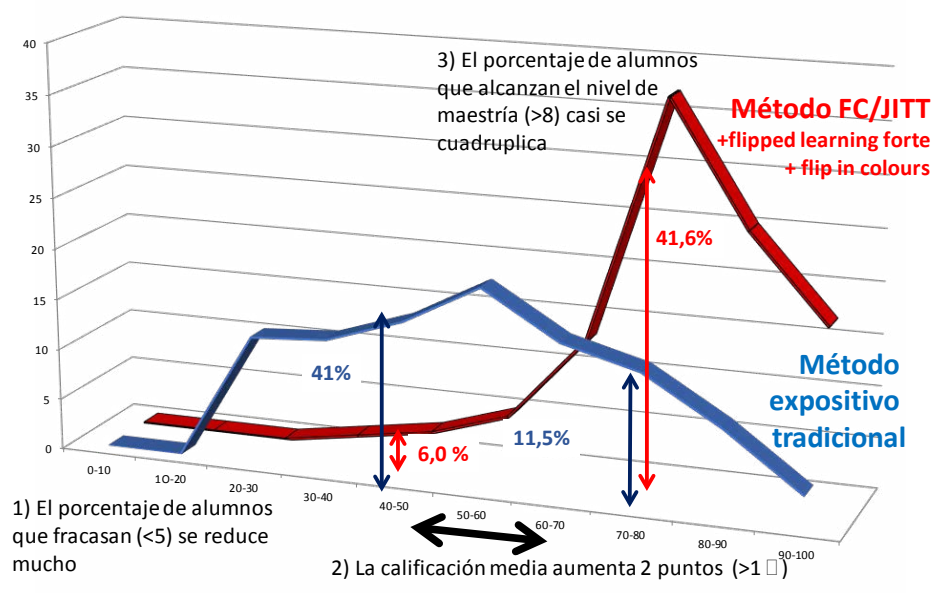

El empleo de estos dos métodos, también nos ha permitido mejorar sensiblemente la percepción de los alumnos sobre la labor de los profesores. Cuando se usa el modelo de aprendizaje inverso en ocasiones parte de los alumnos pueden sentir que se les está obligando a trabajar más de lo debido y que por ello algunos alumnos se enfadan y castigan a sus profesores en las encuestas que recogen su opinión sobre la docencia. El uso del método del flipped learning forte con su envío semanal de respuestas a las dudas urgentes de sus alumnos hace que estos perciban de una manera muy directa, cercana e intensa los esfuerzos del profesor por ayudarles a comprender la materia y superar sus dudas. Esta percepción de la labor de ayuda del profesor se manifiesta en una mejora significativa en las puntuaciones obtenidas por los profesores en las encuestas de opinión de los alumnos sobre la docencia. En el caso del flip in colours también deja claro a los alumnos que el profesor se preocupa de resolver sus dudas, trabajándolas en clase con nuevas actividades diseñadas precisamente para ello. 


\section{CÓMO SE EXPLICA EL IMPACTO TAN POSITIVO DEL MODELO DE AULA INVERSA SOBRE EL APRENDIZAJE}

Cabe preguntarse sobre cuáles son las razones que explican la eficacia de esta combinación de métodos. La primera razón es que este modelo de aprendizaje inverso fomenta el estudio previo de nuestros alumnos con gran eficacia y permite al profesor verificar el estudio y premiar a aquellos que lo hacen, reforzándoles. En algunas asignaturas se ha conseguido que el alumno medio que realiza el estudio previo dedique unas dos horas semanales al estudio previo de los temas que supone hasta unas 30 horas de trabajo per capita de cada alumno por cuatrimestre y asignatura en la que se usa esta metodología.

El sistema de marketing del método y de gamificación de la participación en el mismo son eficaces pues contribuyen a lograr implicar a casi la totalidad de los alumnos a estudiar la mayoría de los temas. Como prueba de esta relación entre estudio previo y mejora de los resultados de aprendizaje, los datos demuestran correlación entre el porcentaje de alumnos que realizan el estudio previo de al menos la mitad de los temas y la mejora en las calificaciones medias obtenidas en las pruebas de evaluación del aprendizaje. Además, el estudio preparatorio crea las condiciones ideales para una interacción presencial de calidad y altamente interactiva.

Este modelo impone al profesor y a sus alumnos fechas límite para realizar determinadas tareas (figura 6). En primer lugar, el profesor se obliga a enviar los temas con una semana de antelación a su tratamiento en clase y esto le hace revisar las futuras clases con más antelación que el repaso de la víspera. En segundo lugar, impone al alumno las tareas de estudiar y reflexionar y enviar sus respuestas al profesor. La recepción de las respuestas de los alumnos presiona al profesor para que las analice y proporcione feedback anticipado a los alumnos y modificaciones al plan de clase. Finalmente, el feedback del profesor también se convierte en materia que debe ser revisada por los alumnos. 
Figura 6. El flipped learning forte mejora el aprendizaje porque impone varias fechas límite y hace necesario realizar a sus alumnos y a sus profesores una serie de tareas que mejoran el aprendizaje. Las líneas verticales gruesas representan las fechas límite para el profesor (negra continua) y para los alumnos (discontinua)

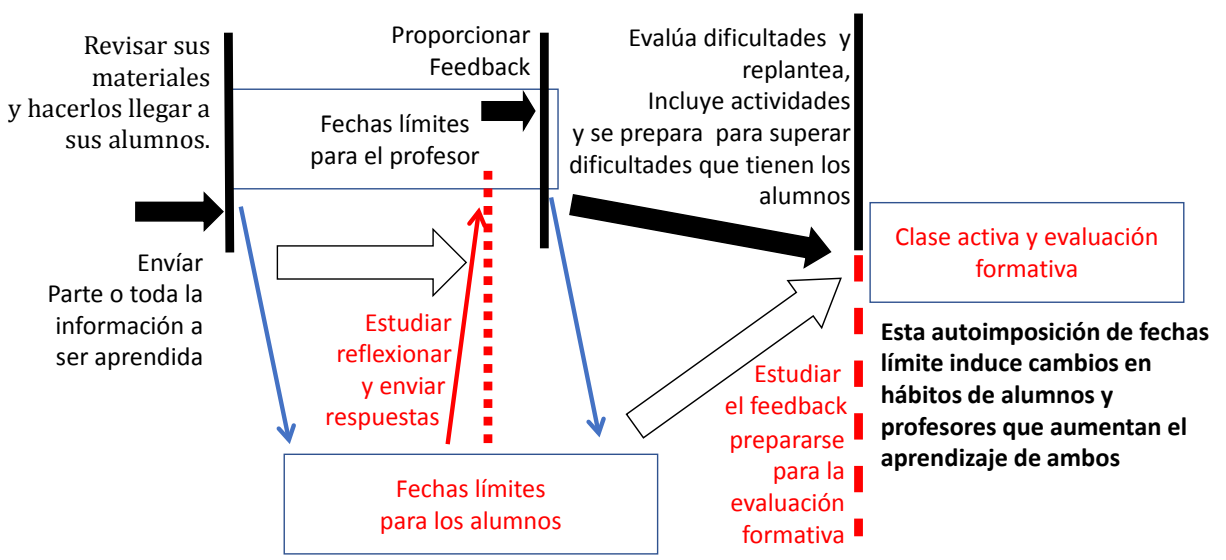

Este modelo hace que muchos alumnos cambien sus hábitos de estudio y participación en clase y ofrece más oportunidades para que los alumnos practiquen en clase bajo la supervisión de su profesor. Las tareas de evaluación formativa en clase estimulan a los alumnos para que repasen y les entrenan para que mejoren sus resultados en las pruebas de evaluación sumativa. Todo esto mejora la comprensión de las ideas esenciales lo que repercute positivamente en su retención a largo plazo y en su capacidad de transferencia a nuevas situaciones.

Por otro lado, el modelo flipped es eficaz en coste de trabajo para el profesor, pues los cuestionarios online y el tratamiento de las respuestas en matrices de datos ahorran mucho trabajo al profesor que puede realizar un análisis de respuestas de sus alumnos para proporcionarles feedback y ajustar sus planes de clase a sus intereses y dificultades.

El modelo flippped tiene otras ventajas interesantes pues fomenta la interacción del alumno con los materiales, la comunicación individual con su profesor y que este proporcione a sus alumnos feedback colectivo que es muy valorado por ellos. Las respuestas de los alumnos también aportan información muy valiosa para que el profesor replantee como usar el tiempo de clase de aquellas maneras que más puedan ayudar a que los alumnos logren conseguir los resultados de aprendizaje programados. 


\section{REFERENCIAS BIBLIOGRÁFICAS}

Amo, D., y Santiago, R. (2017). Learning Analytics La narración del aprendizaje a través de los datos. Barcelona: Universitat Oberta de Catalunya.

Angelo, T., y Cross, P. (1993). Classroom assessment techniques. San Francisco: Jossey-Bass.

Bain, K. (2004). What the best college students do. Cambridge: Harvard University Press.

Barjola, P. et al. (2011). Crédito ECTS: ¿Realidad o Ficción? Bordón, 63(2), 75-90.

Biggs, J. (2006). Calidad del aprendizaje universitario. Madrid: Narcea.

Bloom, B. S., Englehart, M. D., Furst, E. J., y Krathwohl, D. R. (1956). Taxonomy of educational objectives: Cognitive domain. New York: McKay.

Chou, Y. (2015). Actionable Gamification: Beyond Points. Badges, and Leaderboards, Kindle Edition Octalysis Media.

Churches, A. (2007). Edorigami, Blooms taxonomy and digital approaches. [Wiki]. Recuperado de http://edorigami.wikispaces. com/Bloom\%27S+and+ICT+tools

Gómez-Esquer, F. et al. (2011). Cálculo del volumen del trabajo del alumno en las nuevas titulaciones de grado en ciencias de la salud en Evaluación global de los resultados del aprendizaje en las titulaciones dentro del EEES. Madrid: Dyckinson.

Freeman, S. et al. (2014). Active learning increases student performance in Science, Engineering, and Mathematics. Proc Natl Acad Sci US A. 111(23), 8410-8415.

Knight, J., y Wood, W. B. (2005). Teaching more by lecturing less Cell Biol Educ. Winter, 4(4), 298-310.

Huston, T. (2012). Teaching what you don't know. Cambridge: Harvard University Press.

Mazur, E. (1997). Peer instruction: A User's Manual. Prentice Hall Series in Educational Innovation. Englewood Cliffs, NJ: Prentice Hall.
Medina-Moya, J. L. et al. (2016). La docencia universitaria mediante el enfoque del aula invertida. Barcelona: Octaedro.

Michaelsen, L. K., Knight, A. B., y Fink, L. D. (2002). Team-Based Learning: A Transformative Use of Small Groups in College. Westport: Praeger Publishers.

Novak, G., Gavrin, A., Christian, W., y Patterson, E. (1999). Just-In-Time Teaching: Blending Active Learning with Web Technology. Upper Saddle River. NJ: Prentice-Hall.

Markzewski, A. (2013a). Gamification: A Simple Introduction \& a Bit More Kindle edition.

Markzewski, A. (2013b). Thin Layer vs Deep Level Gamification. [Blog Gamification UK]. Recuperado de http://marczewski. me.uk/2013/12/23/thin-layer-vs-deeplevel-gamification/\#.Uzmkxah vvh

McTighe, J., y Wiggins, G. (2013). Essential questions: opening doors to student understanding. Alexandria: ASCD.

Michael, J. (2006). Where's the evidence that active learning Works. Adv Physiol Educ, 30, 159-167.

Prieto, A. et al. (2006). Un nuevo modelo de aprendizaje basado en problemas, el ABP 4X4 es eficaz para desarrollar competencias profesionales valiosas en asignaturas con más de 100 alumnos. Aula Abierta 87, 171194.

Prieto, A., Díaz D., Monserrat J., y Reyes E. (2014a). Experiencias de aplicación de estrategias de gamificación a entornos de aprendizaje universitario. Revisión, 7(2), 7692.

Prieto, A. (2014b). ¿Trabajan en su aprendizaje nuestros alumnos universitarios lo que deberían? Profesor 3.O. Recuperado de http://profesor3puntoo.blogspot.com. es/2013/05/sobre-cerditos-y-creditos-ectsel.html

Prieto, A., Díaz, D., y Santiago, R. (2015). Metodologías Inductivas: El desafio de enseñar mediante el cuestionamiento y los retos. Barcelona: Océano. 
Prieto, A., y Velez, J. (2016). Registro de profesores que usan el modelo de aprendizaje inverso (flipped learning) en países de habla hispana [cuestionario online]. Recuperado de https://goo.gl/ forms/wHaXdd5CvIDNoKrs2

Prince, M. (2004). Does active learning work? J. Engr. Education, 93(3), 223-231.

Robles, G., González-Barahona, J. M., y Prieto, A. (2010). Fomentando la preparación de clase por parte de los alumnos mediante el Campus Virtual. Relada, 4(3), 240-248. Recuperado de http://polired.upm.es/ index.php/relada/article/viewFile/117/113

Sams, A., y Bergmann, J. (2012). Flip your Classroom: Reach every student in every class every day. ISTE.
Sams, A., y Bergmann, J. (2014). Flipped learning gateway to student engagement. International Society for Technology in Education.

Sheldon, L. (2012). The Multiplayer Classroom: Designing Coursework as a Game. Boston: Cengage Learning.

Svinicki, M. (2004). Learning and motivation in the postsecondary classroom. Bolton: Anker Publishing.

Touron, J., Santiago, R., y Diez, A. (2014). The Flipped Classroom: Cómo convertir la escuela en un espacio de aprendizaje. Barcelona: Océano.

Weimer, M. (2013). Learner centered teaching. San Francisco: John Willey \& Sons.

\section{PERFIL ACADÉMICO Y PROFESIONAL DE LOS AUTORES}

Alfredo Prieto Martín. Es profesor contratado. Doctor del Departamento de Medicina y Especialidades Médicas de la Universidad de Alcalá. Premio al mejor investigador Joven de la Universidad de Alcalá 2000. Premio innovación docente 2014 Universidad de Alcalá. Profesor de formación del profesorado en 26 universidades españolas. Autor del Blog Profesor 3.0 http://profesor3puntoo. blogspot.com.es

E-mail:alfredo.prieto@uah.es

\section{DIRECCIÓN DEL AUTOR}

Departamento de Medicina y Especialidades Médicas

Universidad de Alcalá. España

Fecha de recepción del artículo: 20/04/2017

Fecha de aceptación del artículo: 23/06/2017

\section{Como citar este artículo:}

Prieto Martín, A., Díaz Martin, D., Aguilera, I. L., Monserrat Sanz, J., Sanvicen Torner, P., Santiago Campión, R., Corell Almuzara, A., y Álvarez-Mon Soto, M. (2018). Nuevas combinaciones de aula inversa con just in time teaching y análisis de respuestas de los alumnos. RIED. Revista Iberoamericana de Educación a Distancia, 21(1), pp. 175-194. doi: http://dx.doi.org/10.5944/ried.21.1.18836 